\title{
Recessive optic atrophy, sensorimotor neuropathy and cataract associated with novel compound heterozygous mutations in OPAI
}

\author{
JINHO LEE $^{1 *}$, SUNG-CHUL JUNG $^{2 *}$, YOUNG BIN HONG ${ }^{1}$, JEONG HYUN YOO $^{3}$, HEASOO KOO $^{4}$, \\ JA HYUN LEE ${ }^{5}$, HYUN DAE HONG ${ }^{5}$, SANG-BEOM KIM ${ }^{6}$, KI WHA CHUNG ${ }^{5 *}$ and BYUNG-OK CHOI ${ }^{*}$ \\ ${ }^{1}$ Department of Neurology, Samsung Medical Center, Sungkyunkwan University School of Medicine, Seoul 135-710; \\ Departments of ${ }^{2}$ Biochemistry, ${ }^{3}$ Radiology and ${ }^{4}$ Pathology, Ewha Womans University School of Medicine, \\ Seoul 120-750; ${ }^{5}$ Department of Biological Science, Kongju National University, Konju, Chungnam 314-701; \\ ${ }^{6}$ Department of Neurology, Kyung Hee University College of Medicine, \\ Seoul 143-601, Republic of Korea
}

Received February 3, 2015; Accepted November 30, 2015

DOI: $10.3892 / \mathrm{mmr} .2016 .5209$

\begin{abstract}
Mutations in the optic atrophy 1 gene (OPAl) are associated with autosomal dominant optic atrophy and $20 \%$ of patients demonstrate extra-ocular manifestations. In addition to these autosomal dominant cases, only a few syndromic cases have been reported thus far with compound heterozygous OPA1 mutations, suggestive of either recessive or semi-dominant patterns of inheritance. The majority of these patients were diagnosed with Behr syndrome, characterized by optic atrophy, ataxia and peripheral neuropathy. The present study describes a 10 -year-old boy with Behr syndrome presenting with early-onset severe optic atrophy, sensorimotor neuropathy, ataxia and congenital cataracts. He had optic atrophy and was declared legally blind at six years old. Electrophysiological, radiological, and histopathological findings were compatible with axonal sensorimotor polyneuropathy. At birth, he presented with a congenital cataract, which has not been previously described in patients with OPA1 mutations. Whole exome sequencing indicated a pair of novel compound heterozygous mutations: p.L620fs*13 (c.1857-1858delinsT) and p.R905Q (c.G2714A). Neither mutation was observed in controls $(n=300)$, and thus, they were
\end{abstract}

Correspondence to: Professor Byung-Ok Choi, Department of Neurology, Samsung Medical Center, Sungkyunkwan University School of Medicine, 81 Irwon-ro, Seoul 135-710, Republic of Korea E-mail: bochoi77@hanmail.net

Professor Ki Wha Chung, Department of Biological Science, Kongju National University, 56 Gonjudaehak-ro, Konju, Chungnam 314-701, Republic of Korea

E-mail: kwchung@kongju.ac.kr

*Contributed equally

Key words: recessive optic atrophy, Behr syndrome, congenital cataracts, the optic atrophy 1 gene, compound heterozygous OPAI mutations predicted to be pathogenic by multiple in silico analyses. The mutation sites were highly conserved throughout different vertebrate species. The patients parents did not have any ophthalmic or neurologic symptoms and the results of electrophysiological studies were normal, suggestive of an autosomal recessive pattern of inheritance. The present study identified novel compound heterozygous $O P A 1$ mutations in a patient with recessive optic atrophy, sensorimotor neuropathy and congenital cataracts, indicating an expansion of the clinical spectrum of pathologies associated with OPAl mutations. Thus, OPAl gene screening is advisable in the workup of patients with recessive optic atrophy, particularly with Behr syndrome and cataracts.

\section{Introduction}

Optic atrophy 1 gene $(O P A l)$ is a multifunctional protein located within the mitochondrial inner membrane, which regulates a number of critical cellular functions, including mitochondrial fusion, mitochondrial DNA maintenance and apoptosis (1). OPAl belongs to the dynamin family with which it shares three conserved regions, a GTPase domain, a middle domain, and a GTPase effector domain (2). Mutations in the OPA1 gene (3q28-q29, OMIM 165500) are known to result in autosomal dominant optic atrophy (DOA, OMIM 165500), which is the most commonly diagnosed inherited optic neuropathy in clinical practice (2). Up to $20 \%$ of OPAl mutation carriers develop phenotypes that have been designated as DOA+, where the optic atrophy is complicated by a wide range of neuromuscular features, including ataxia, myopathy, peripheral neuropathy, sensorineural deafness, and notably, chronic progressive external ophthalmoplegia (3). With the exception of these autosomal dominant forms, only a few syndromic cases have so far been reported with compound heterozygous $O P A 1$ mutations suggestive of either recessive or semi-dominant patterns of inheritance (3-6). However, the clinical spectrum of these emerging double-mutant $O P A 1$-associated disorders remains to be elucidated.

The majority of patients with $O P A 1$ mutations possess a simple heterozygous mutation. However, only seven 
patients have been genetically confirmed with compound heterozygous mutations and, of these, six were diagnosed with Behr syndrome (OMIM 210000), which is characterized by early-onset optic atrophy, ataxia, pyramidal signs, peripheral neuropathy, mental retardation and developmental delay (7-8). Although it has been demonstrated to be inherited in an autosomal recessive manner, simple heterozygous OPAl mutations have also been reported to result in Behr syndrome (9). Although it is understood to be inherited in an autosomal recessive manner, it had been reported autosomal dominant inheritance.

To date, 35 OPAl mutations have been identified in multiple domains (3), and it is hypothesized that the final common pathway of $O P A 1$ results in retinal ganglion cell loss and optic nerve degeneration, in addition to more widespread neuronal loss with multi-systemic manifestations. In the present study, the detailed clinical manifestations of autosomal recessive optic atrophy, sensorimotor neuropathy and cataracts associated with the novel compound heterozygous mutations in the OPAl gene was reported, with the aims of expand the clinical spectrum of $O P A 1$ mutations.

\section{Patients and methods}

Patients. The present study included a total of three individuals of Korean origin; one patient with optic atrophy and his parents who exhibited the normal phenotype. Clinical characteristics are presented in Table I. Paternity was determined by genotyping 15 microsatellites using a PowerPlex 16 system (Promega Corporation, Madison, WI, USA). In addition to this family, 300 healthy individuals were examined as controls. Written informed consent was obtained from all participants according to a protocol approved by the Institutional Review Board for Sungkyunkwan University, Samsung Medical Center (Seoul, South Korea), and Kongju National University (Chungnam, South Korea; Industry-Cooperation Foundation).

Whole exome sequencing (WES) and determination of causative mutations. Peripheral blood DNA was isolated using a QIAamp blood DNA purification kit (Qiagen $\mathrm{GmbH}$, Hilden, Germany). Exome sequencing has been recently introduced to inherited peripheral neuropathy as an efficient molecular diagnostic tool (10). WES was performed using the SeqCap EZ Human Exome Library version 3.0 (Roche NimbleGen, Inc., Madison, WI, USA), and the HiSeq 2000 Genome Analyzer (Illumina, Inc., San Diego, CA, USA) for the three individuals (designated I-1, I-2, and II-1 in the FC394 family) using a previously described method (10). The UCSC assembly hg19 (NCBI build 37.1) was used as the reference sequence. In the present study variants with $>20$ single nucleotide polymorphism (SNP) quality were used. Functionally significant variants (missense, nonsense, exonic indel and splicing site variants) were selected from hereditary peripheral neuropathy- and optic atrophy-associated genes, and these were then compared with the dbSNP142 database (http://www.ncbi.nlm.nih.gov), the 1,000 Genomes Project database (http://www.1000genomes.org/) and the Exome Variant Project (http://evs.gs.washington.edu/EVS/).

Candidate causative variants were confirmed by Sanger sequencing for the three individuals using an ABI3130XL
Genetic Analyzer (Applied Biosystems; Thermo Fisher Scientific, Inc., Waltham, MA, USA). Mutations were considered causative when they: (i) Were inherited in a recessive pattern or occurred by de novo mutation; (ii) co-segregated with affected members; (iii) were not found in any of the 300 healthy controls; and (iv) were not reported in the dbSNP142 database, the 1000 Genomes Project database or the Exome Variant Project. Genotype-phenotype correlation was also considered for candidate variants. Genomic evolutionary rate profiling (GERP) scores were determined by the GERP++ program (http://mendel.stanford.edu/SidowLab/downloads/gerp/index.html). In silico analyses were performed using the prediction algorithms of SIFT (http://sift.jcvi.org), MUpro (http://www.ics.uci.edu/ baldig/mutation), and PolyPhen2 (http://genetics.bwh.harvard.edu/pph2/).

Clinical and electrophysiological assessments. All subjects were comprehensively assessed by a team of experienced neurologists and ophthalmologists in order to determine the extent and severity of their ophthalmic, auditory and neurological deficits. Ophthalmic examination included fundoscopic examination, color vision discrimination and automated visual field assessment. Patients were examined for motor and sensory impairment, deep tendon reflexes and muscle atrophy by two independent neurologists, and pure-tone audiometric evaluation was performed.

Nerve conduction studies were performed on all extremities of the patient (Medtronic Keypoint 4; Medtronic Inc., Minneapolis, MN, USA). Motor nerve conduction velocities (MNCVs) of the median and ulnar nerves were determined by stimulation at the elbow or wrist, while recording compound muscle action potentials (CMAPs) over the abductor pollicis brevis and adductor digiti quinti, respectively. Similarly, the MNCVs of the peroneal and tibial nerves were determined by stimulation at the knee and ankle, while recording CMAPs over the extensor digitorum brevis and adductor hallucis, respectively. CMAP amplitudes were measured from baseline to negative peak values. Sensory nerve conduction velocities were obtained over the finger-wrist segment from the median and ulnar nerves by orthodromic scoring, and were also recorded for sural nerves. Sensory nerve action potential (SNAP) amplitudes were measured from positive peaks to negative peaks.

Magnetic resonance imaging (MRI) scans of lower limbs and brain. On a follow-up visit at 8 years old, the patient (II-1) was evaluated using a 3.0-T system (Siemens AG, Munich, Germany). Lower limb imaging was obtained in the axial [field of view (FOV), 24-32 cm; slice thickness, $6 \mathrm{~mm}$; and slice gap, 0.5-1.0 mm] and coronal planes (FOV, 38-40 cm; slice thickness, 4-5 mm; slice gap, 0.5-1.0 mm). The following protocol was used: T1-weighted spin-echo (SE) [repetition time (TR)/echo time (TE) 570-650/14-20, 512 matrices], T2-weighted SE (TR/TE 2800-4000/96-99, 512 matrices), and fat-suppressed T2-weighted SE (TR/TE 3090-4900/85-99, 512 matrices).

Sural nerve biopsy. Histopathological analysis of the distal sural nerve biopsy was performed on the patient (II-1) at 6 years of age. In addition to light microscopic examination (BX51; Olympus Corporation, Tokyo, Japan) of formalin-fixed 


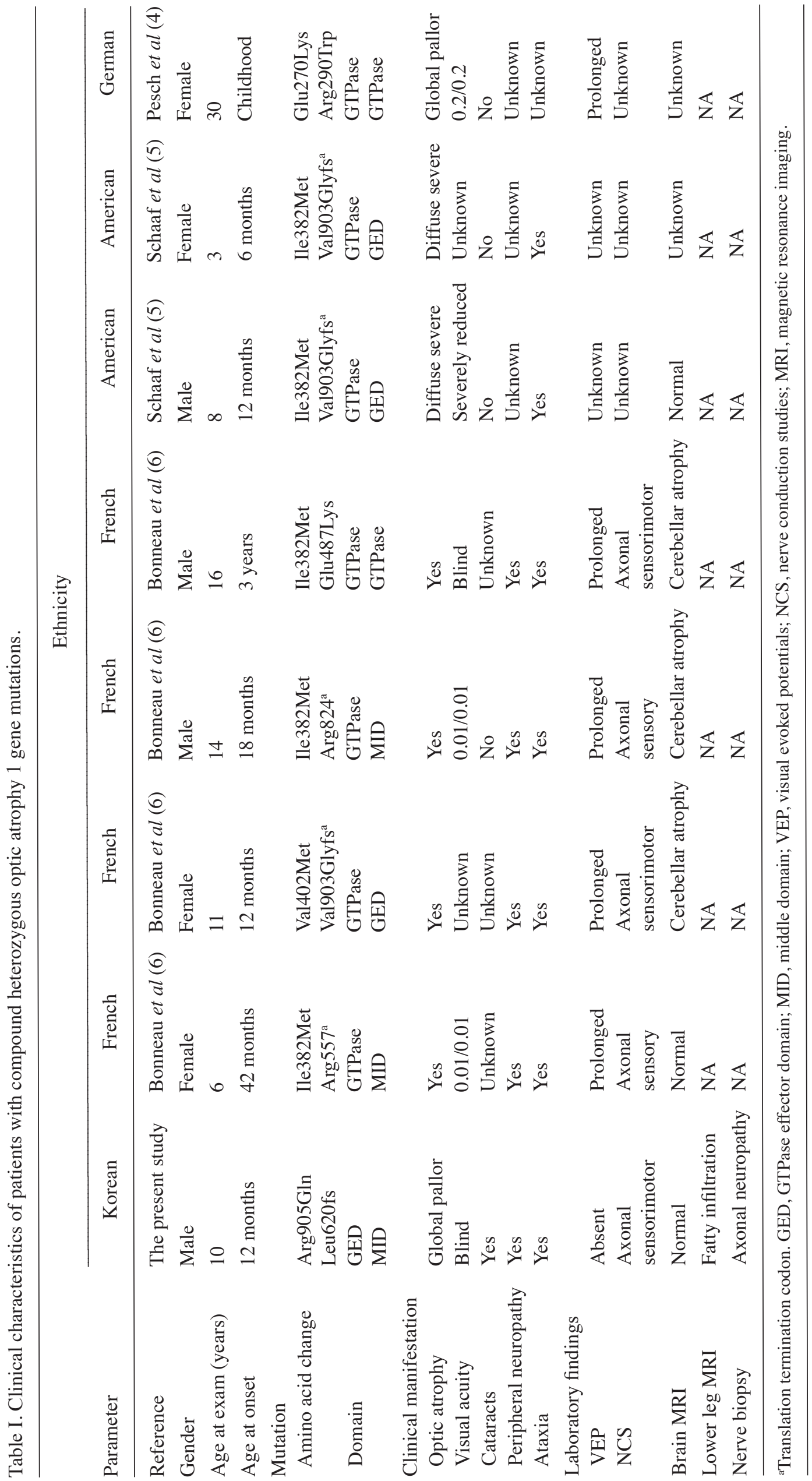


Table II. Whole exome sequencing analysis of the proband and his parents.

\begin{tabular}{lccc}
\hline Items/samples & I-1 (father) & I-2 (mother) & II-1 (patient) \\
\hline Total yield (Gbp) & 14,27 & 9,48 & 5,30 \\
Mappable reads (\%) & 99.2 & 99.4 & 92.1 \\
Coverage of the target region ( $\geq 1 \mathrm{X}, \%)$ & 95.7 & 95.2 & 97.9 \\
Coverage of the target region ( $\geq 10 \mathrm{X}, \%)$ & 90.5 & 88.2 & 92.9 \\
Mean read depth of the target region (X) & 58.7 & 40.6 & 57.9 \\
Total number of SNPs & 86,313 & 84,206 & 49,686 \\
Number of coding SNPs & 20,265 & 20,498 & 19,472 \\
Total number of indels & 7,902 & 7,472 & 6,898 \\
Number of coding indels & 390 & 398 & 508
\end{tabular}

SNP, single nucleotide polymorphism.

A

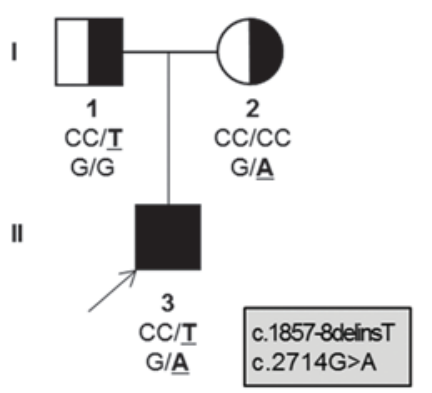

C

Homo sapiens.
Mus musculus
Rattus norvegicus
Bostaurus
Gallusgallus
Xenopus(Silurana) tropicalis
Danio rerio

B

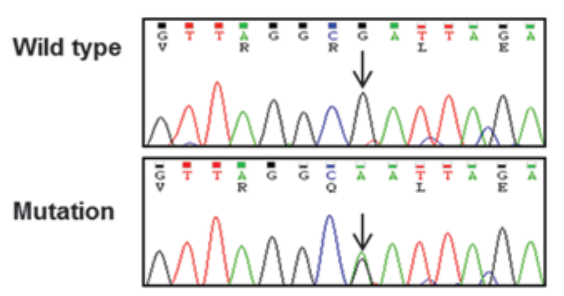

c.1857-1858delins T

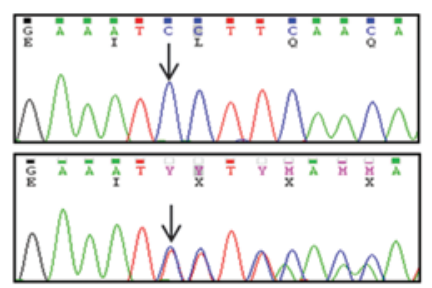

p.R905Q

QQLTNTEVRRLEKNVKEV VTPKHWEEILQQSLWERV

QQLTNTEVRRLEKNVKEV VTPKHWEEILQQSLWERV

QQLTNTEVRRLEKNVKEV VTPKHWEEILQQSLWERV

QQLTNTEVRRLEKNVKEV VTPKHWEEILQQSLWERV

QQLTNTEVRRLEKNVKEV VTPKHWEE I LQKTLWERV

QQL INTEVRRLEKNVKEV VTPKHWEEI LQRTMWERV

QQLTNTEVRRLEKNVKEV VTPKHWESILQKKLWERV

Figure 1. Pedigree and sequencing of the presented family and sequence conservation. (A) Pedigree, half-filled symbols indicate unaffected carriers with one heterozygous mutation, and filled symbols indicate affected individuals. The arrow indicates the proband. Genotypes of both $O P A 1$ mutations are indicated below each examined individual. (B) Sequencing chromatograms, vertical arrows indicate the mutation site. (C) Conservation analysis of amino acid sequences; mutation sites and neighboring sequences were well conserved across vertebrate species (Homo sapiens: NP_056375.2, Mus musculus: NP_598513.1, Rattus norvegicus: NP_598269.3, Bos taurus: NP_001179890.1, Gallus gallus: NP_001034398.1, Xenopus (Silurana) tropicalis: NP_001120510.1, and Danio rerio: NP_001007299.1).

sections, electron microscopic observations were made using specimens fixed in $2 \%$ glutaraldehyde in $0.025 \mathrm{M}$ cacodylate buffer (pH 7.4; both Sigma-Aldrich, St. Louis, MA, USA) and processed for semi-thin and ultra-thin studies. Semi-thin sections were stained with toluidine blue (Sigma-Aldrich) for evaluation under light microscopy. Ultra-thin sections (60-65 nm) were contrasted with uranyl acetate and lead citrate (both Sigma-Aldrich) for ultrastructural studies (H-7650; Hitachi, Ltd., Tokyo, Japan). The density of myelinated fibers (MFs), axonal diameter, myelin thickness, and the g-ratio of MFs were determined from semi-thin transverse sections using a computer-assisted image analyzer (AnalySIS; Olympus Soft Imaging Solutions, GmbH, Münster, Germany).

\section{Results}

Identification of compound heterozygous mutations in OPA1. Based on WES in the father-mother-patient trio
(Table II; Fig. 1A), the patient possessed a pair of novel compound heterozygous mutations in $O P A 1$, p.L620fs" 13 (c.1857-1858delinsT) in exon 20 and p.R905Q (c.G2714A) in exon 27 (Fig. 1B). These mutations were located in the middle domain and the guanosine triphosphatase (GTPase) effector domain, respectively. Parental analysis indicated that the mother was heterozygous for the p.R905Q missense mutation and the father was heterozygous for the p.L620fs frameshift mutation. Neither mutation was detected in the 300 healthy Korean controls, dbSNP138 (http://www.ncbi.nlm.nih.gov), the 1,000 Genomes Database (http://www.1000genomes. org/) or the Exome Variant Server (http://evs.gs.washington. edu/EVS/). Amino acid sequences surrounding the mutation sites were highly conserved throughout different vertebrate species (Fig. 1C). Three in silico analyses (SIFT, PolyPhen2 and MUpro) indicated pathogenic prediction (Table III). The GERP scores of the mutations were high (5.650 at c.1858C and 5.890 at c.2714G). Thus, these novel compound hetero- 
Table III. Summary of optic atrophy 1 gene mutations and in silico scores.

\begin{tabular}{lcccccr}
\hline & \multirow{2}{*}{$\begin{array}{c}\text { Nucleotide } \\
\text { change }\end{array}$} & $\begin{array}{c}\text { Amino acid } \\
\text { change }\end{array}$ & SIFT & PolyPhen2 & MUpro & $\begin{array}{c}\text { GERP } \\
\text { score }\end{array}$ \\
\cline { 5 - 6 } MID & c.1857-1858delinsT & L620fs*13 & - & - & - & 5.650 \\
GED & c.2714G $>$ A & R905Q & $0.000^{\mathrm{c}}$ & $1.000^{\mathrm{c}}$ & $-0.630^{\mathrm{c}}$ & 5.890 \\
\hline
\end{tabular}

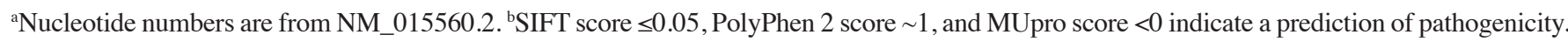
'Denotes a 'pathogenic' prediction. MID, middle domain; GED, GTPase effector domain; GERP, genomic evolutionary rate profiling score.
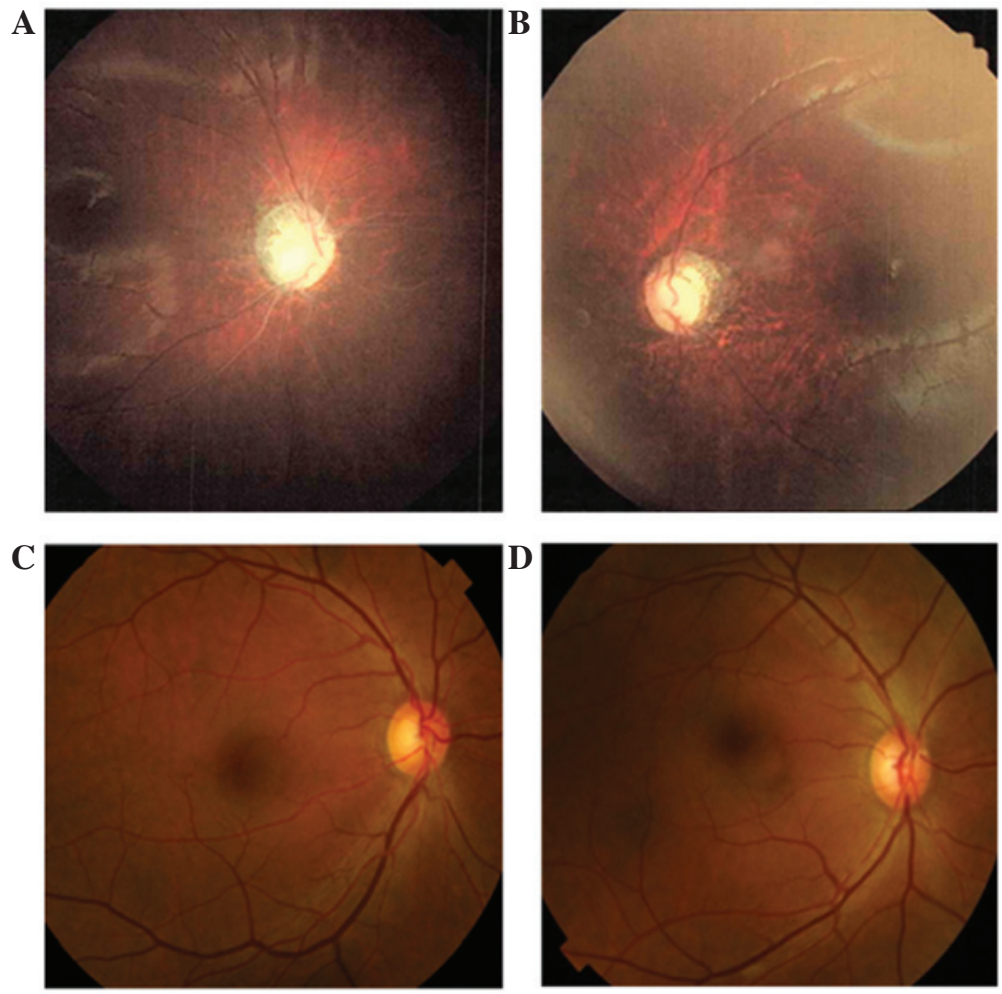

Figure 2. Fundoscopic examination of the presented family. Proband (II-1) showed globally pale optic discs in his (A) right and (B) left eye, however, his (C) father and (D) mother had normal optic discs.

zygous mutations in the OPAl gene were likely the underlying cause of the patient's phenotype.

In addition to the OPAI causative mutations, multiple functionally notable variants were detected in optic atrophy and hereditary peripheral neuropathy associated genes. However, these were considered polymorphic or rare private variants, as they were observed in controls, including various human genome variants databases. Multiple variants were not observed in the controls, but they did not co-segregate with the affected individual in the present family.

Clinical manifestations. Clinical manifestations are summarized in Table I. A 10-year-old boy (II-1; Fig. 1A), was born healthy at full term by spontaneous vaginal delivery to healthy non-consanguineous Korean parents. He visited the Department of Neurology at Samsung Medical Center (Seoul, Korea) aged 10 years due to gait disturbance. He was diagnosed with congenital cataracts at the age of one and was treated with a corrective operation; at that point, optic atrophy and nystagmus were noted in his eyes. The optic atrophy progressed to the point that he was declared legally blind at six years of age. By age two, he was not able to walk without support, and by the age of 10 , he harbored severe ataxia and postural instability. Neurological examination at 10 years of age indicated muscle weakness and atrophy of the bilateral distal muscles, predominantly in the lower limbs. Bilateral pes cavus, steppage gait, and atrophic changes of the intrinsic foot and calf muscles were noted. Sensitivity to pinprick, touch, position, and vibration was decreased. Knee and ankle jerks were absent and pyramidal and cerebellar signs were not observed. At 10 years of age, the patient was not able to count fingers at $\sim 15 \mathrm{~cm}$ with either eye but did perceive light. A dilated fundus examination demonstrated diffuse severe optic atrophy in his eyes (Fig. 2A and B). Audiological evaluation indicated normal hearing sensitivity. Laboratory tests, such as glucose level, liver enzymes, and renal function were normal, and echocardiography indicated a normal sinus rhythm. Although 
Table IV. Serial electrophysiological studies in a patient (II-1) with novel compound mutations in the optic atrophy 1 gene.

\begin{tabular}{|c|c|c|c|c|c|c|c|}
\hline \multirow[b]{2}{*}{ Parameter } & \multicolumn{2}{|c|}{ Side $^{a}$} & \multicolumn{2}{|c|}{ Side $^{b}$} & \multicolumn{2}{|c|}{ Side $^{c}$} & \multirow[b]{2}{*}{ Normal value } \\
\hline & Right & Left & Right & Left & Right & Left & \\
\hline \multicolumn{8}{|l|}{ Median nerve } \\
\hline $\mathrm{TL}(\mathrm{ms})$ & 3.0 & 2.8 & 3.4 & 2.9 & 3.3 & 3.0 & $<3.9$ \\
\hline CMAP (mV) & 7.9 & 8.8 & 7.3 & 6.9 & 6.2 & 7.6 & $>6.0$ \\
\hline $\operatorname{MNCV}(\mathrm{m} / \mathrm{sec})$ & 54.2 & 54.2 & 51.8 & 53.7 & 48.0 & 55.0 & $>50.5$ \\
\hline \multicolumn{8}{|l|}{ Ulnar nerve } \\
\hline $\mathrm{TL}(\mathrm{ms})$ & 2.4 & 2.4 & 2.4 & 2.8 & 2.8 & 2.6 & $<3.0$ \\
\hline CMAP $(\mathrm{mV})$ & 8.0 & 6.1 & 7.4 & 6.7 & 8.1 & 7.4 & $>8.0$ \\
\hline $\operatorname{MNCV}(\mathrm{m} / \mathrm{sec})$ & 53.8 & 54.0 & 53.6 & 53.4 & 55.0 & 53.0 & $>51.1$ \\
\hline \multicolumn{8}{|l|}{ Peroneal nerve } \\
\hline $\mathrm{TL}(\mathrm{ms})$ & 2.3 & 2.9 & 2.7 & 3.0 & 3.8 & 3.6 & $<5.3$ \\
\hline CMAP (mV) & 1.1 & 1.3 & 1.1 & 1.0 & 0.6 & 1.7 & $>1.6$ \\
\hline $\operatorname{MNCV}(\mathrm{m} / \mathrm{sec})$ & 46.3 & 48.6 & 42.2 & 43.5 & 45.0 & 50.0 & $>41.2$ \\
\hline \multicolumn{8}{|l|}{ Tibial nerve } \\
\hline $\mathrm{TL}(\mathrm{ms})$ & 2.3 & 2.6 & 2.9 & 3.0 & 4.1 & 3.9 & $<5.4$ \\
\hline CMAP (mV) & 4.7 & 5.1 & 2.6 & 2.8 & 2.9 & 2.2 & $>6.0$ \\
\hline $\operatorname{MNCV}(\mathrm{m} / \mathrm{sec})$ & 45.5 & 46.3 & 42.0 & 43.8 & 41.0 & 45.0 & $>41.1$ \\
\hline \multicolumn{8}{|c|}{ Median sensory nerve } \\
\hline SNAP $(\mu \mathrm{V})$ & $\mathbf{A}$ & $\mathbf{A}$ & $\mathbf{A}$ & $\mathbf{A}$ & $\mathbf{A}$ & $\mathbf{A}$ & $>8.8$ \\
\hline $\mathrm{SNCV}(\mathrm{m} / \mathrm{sec})$ & $\mathbf{A}$ & $\mathbf{A}$ & $\mathbf{A}$ & $\mathbf{A}$ & $\mathbf{A}$ & $\mathbf{A}$ & $>39.3$ \\
\hline \multicolumn{8}{|c|}{ Ulnar sensory nerve } \\
\hline SNAP $(\mu \mathrm{V})$ & $\mathbf{A}$ & $\mathbf{A}$ & $\mathbf{A}$ & $\mathbf{A}$ & $\mathbf{A}$ & $\mathbf{A}$ & $>7.9$ \\
\hline $\mathrm{SNCV}(\mathrm{m} / \mathrm{sec})$ & $\mathbf{A}$ & $\mathbf{A}$ & A & $\mathbf{A}$ & $\mathbf{A}$ & $\mathbf{A}$ & $>37.5$ \\
\hline \multicolumn{8}{|l|}{ Sural nerve } \\
\hline $\operatorname{SNAP}(\mu \mathrm{V})$ & $\mathbf{A}$ & $\mathbf{A}$ & $\mathbf{A}$ & $\mathbf{A}$ & $\mathbf{A}$ & $\mathbf{A}$ & $>6.0$ \\
\hline $\mathrm{SNCV}(\mathrm{m} / \mathrm{sec})$ & $\mathbf{A}$ & $\mathbf{A}$ & $\mathbf{A}$ & $\mathbf{A}$ & $\mathbf{A}$ & $\mathbf{A}$ & $>32.1$ \\
\hline
\end{tabular}

${ }^{\mathrm{a}-\mathrm{c}}$ Examination at age 6,8 and 10, respectively. Bold characters indicate abnormal values. A, absent potentials; TL, terminal latency; CMAP, compound muscle action potential; MNCV, motor nerve conduction velocity; SNAP, sensory nerve action potential; SNCV, sensory nerve conduction velocity.

his father and mother had an $O P A l$ heterozygous mutation, no abnormalities following ophthalmological and electrophysiological evaluation were observed (I-1, and I-2; Fig. 1A). They did not complain of any visual problems and dilated fundus examination results, including assessment of each optic nerve and formal color vision test, were normal (Fig. 2C and D).

Electrophysiological features are summarized in Table IV. SNAPs were absent in of the patient at all extremities at 6,8 and 10 years of age. CMAPs were decreased in the peroneal and tibial nerves, compatible with axonal polyneuropathy. When visual evoked potentials (VEP) were performed at 6 years, no potential was demonstrated. However, brainstem auditory evoked potentials (BAEP) were normal. In the parents, VEP and BAEP were normal.

Length-dependent patterns in the lower extremity MRI. At the age of 10 years, T1-weighted MRIs indicated muscle atrophy and fatty replacement in the lower calf muscles but close to normal hip and thigh muscles. These findings are compatible with length-dependent axonal degeneration. In addition, fatty replacement of the calf muscles was predominant in the anterior compartment, including the tibialis anterior muscle, consistent with his foot drop and steppage gait (Fig. 3A-D). A brain MRI performed when he was 8 years old indicated the cerebral cortex and cerebellum were normal in structure and size.

Histopathological findings. Semi-thin transverse sections of the distal sural nerve demonstrated medium and small MFs with complete loss of large MFs. Compared with a healthy distal sural nerve MF count of $14,170 \pm 2,250 / \mathrm{mm}^{2}$ in a normal 2-year-old female, 4,245 MFs $/ \mathrm{mm}^{2}$ were counted (Fig. 4A). The range and average MF diameter were 2.5-10.3 $\mu \mathrm{m}$ and $5.0 \mu \mathrm{m}$, respectively. A histogram indicated a unimodal distribution pattern; MFs with a diameter $<3 \mu$ m comprised $3.6 \%$ of the total population (Fig. 4B), the MFs with a diameter $>8 \mu \mathrm{m}$ comprised $0.9 \%$ of the total population. The MF\% area in this case was $8.8 \%$. The remaining MFs demonstrated occasional excessive folding of myelin, with very rare evidence of regeneration. There was no evidence of demyelination, remyelination, or onion bulb formation. Electron microscopic examination indicated that the 

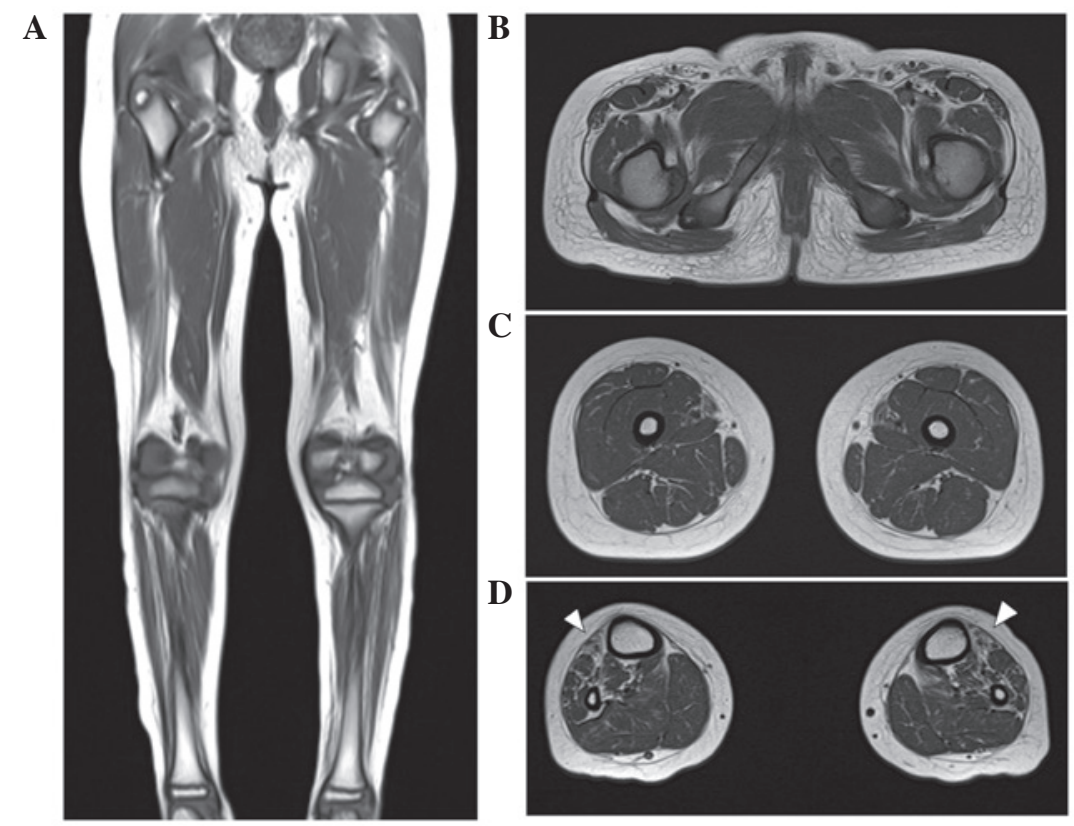

Figure 3. Lower limb MRI of the proband. T1-weighted (A) coronal and (B-D) axial images of a lower limb MRI. (B and C) There was minimal fatty infiltration of the other compartments of the lower legs and no prominent abnormal findings in the hip or thigh muscles. (D) However, fatty infiltration and muscle atrophy (arrowheads) were prominently evident in the anterior compartment of both lower legs. MRI, magnetic resonance imaging.
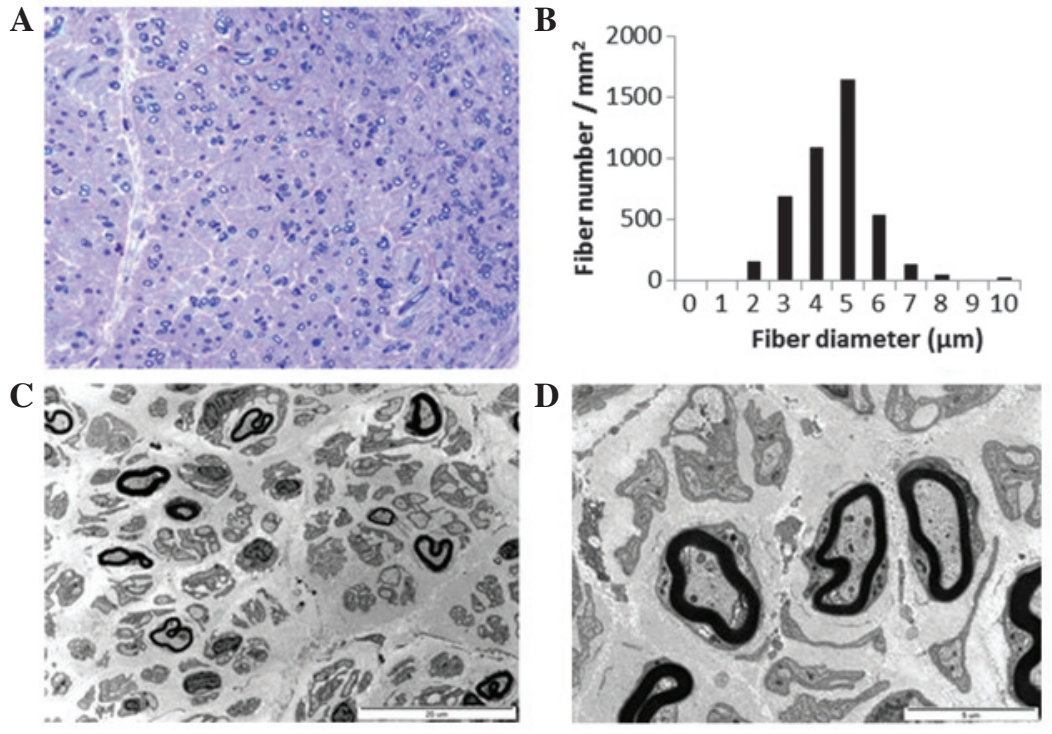

Figure 4. Histopathological findings of the proband. (A) Semi-thin transverse sections revealed almost complete loss of large MFs with preserved medium- and small-sized MFs. Toluidine blue stain; magnification, x400. (B) Histogram demonstrates a unimodal distribution pattern of MFs with a diameter $>8 \mu \mathrm{m}$, which comprised $0.9 \%$ of the total MFs. (C and D) Electron micrographs (magnification, x3,000 and x10,000, respectively) indicated that the remaining MFs had focal abnormalities of myelinated and unmyelinated axons, with occasional rarefaction. MFs, myelinated fibers.

remaining MFs had focal abnormalities of the myelin, including irregular myelin sheaths, distorted shapes or focal noncompaction (Fig. 4C). Unmyelinated axons were relatively well preserved with occasional rarefaction (Fig. 4D). Endoneurial fibroblast proliferation and large quantities of collagen deposition were documented.

\section{Discussion}

Novel compound heterozygous $O P A 1$ mutations were identified in a patient with recessive optic atrophy, sensorimotor neuropathy and congenital cataracts. $O P A l$ is a multifunctional protein located within the mitochondrial inner membrane (1) and mutations in the OPAl gene are known to cause DOA (11). The majority of patients with DOA have isolated optic atrophy and $\sim 20 \%$ of patients present with extra-ocular manifestations, termed DOA+ $(3,12)$. Excluding these autosomal dominant forms, only seven cases have so far been genetically confirmed as compound heterozygous OPAl mutations. The majority of patients with compound heterozygous mutations presented with an earlier onset and more severe disease manifestation, suggestive of either recessive or semi-dominant patterns of 
inheritance (4-6). In the present family, the proband demonstrated early onset severe optic atrophy whereas neither parents had ophthalmic or neurologic symptoms, and the results of electrophysiologic testing, such as nerve conduction studies, VEP and BAEP were normal. These results may support either recessive or semi-dominant inheritance with incomplete penetrance.

Clinical and electrophysiological features of seven cases with confirmed compound heterozygous OPAl mutations were summarized in Table I. Six of them were diagnosed with Behr syndrome except one case in which extra-ocular manifestations were not described. Behr syndrome is characterized by the association of early-onset optic atrophy, ataxia, pyramidal signs, peripheral neuropathy, mental retardation and developmental delay $(7,8)$. However, six patients did not have pyramidal signs, mental retardation or marked developmental delay. All the cases presented with early-onset severe optic atrophy and ataxia. Four patients had peripheral neuropathies and three showed signs of cerebellar atrophy on brain MRIs. Genetically, six of the patients had either one or two OPAl mutations in the GTPase domain. The present case presented with early-onset severe atrophy, sensorimotor neuropathy, and ataxia, and he did not have pyramidal signs, developmental delay or mental retardation, which was similar to previously reported cases (4-6). In addition, he had congenital cataracts, which have not, to the best of our knowledge, been previously described in patients with OPAl mutations. In addition, the patient did not have any mutation in the GTPase domain.

The present case had bilateral severe congenital cataracts, congenital cataracts occur in infants due to various known causes that include inherited conditions, infection, metabolic disorders and ocular diseases $(13,14)$. Inherited conditions are the likely cause of congenital cataracts in this case as there were no specific causes demonstrated by the medical records or laboratory findings, and symptoms were severe and symmetric. Aijaz et al (15) reported that expression of OPAl was observed in the lens and these results may support this assertion. The present case may expand the clinical spectrum of Behr syndrome with compound heterozygous $O P A 1$ mutations.

In conclusion, the present study identified novel compound heterozygous OPAl mutations in a patient with recessive optic atrophy, sensorimotor neuropathy and congenital cataracts. Congenital cataracts that were described in the present case may expand the clinical spectrum in patients with OPAl mutations. Thus, OPAl mutations should be considered when screening patients with recessive optic atrophy, particularly those with peripheral neuropathy, and cataracts.

\section{Acknowledgements}

The present study was supported by the Korean Health Technology R\&D Project, Ministry of Health \& Welfare (grant nos. HI12C0135 and HI14C3484) and by a National Research Foundation of Korea grant funded by the Korean government (MSIP; grant no. 2014R1A2A2A01004240).

\section{References}

1. Lenaers G, Hamel C, Delettre C, Amati-Bonneau P, Procaccio V, Bonneau D, Reynier P and Milea D: Dominant optic atrophy. Orphanet J Rare Dis 7: 46, 2012.

2. Eiberg H, Kjer B, Kjer P and Rosenberg T: Dominant optic atrophy $(O P A 1)$ mapped to chromosome $3 \mathrm{q}$ region. I. Linkage analysis. Hum Mol Genet 3: 977-980, 1994.

3. Yu-Wai-Man P, Griffiths PG, Gorman GS, Lourenco CM, Wright AF, Auer-Grumbach M, Toscano A, Musumeci O, Valentino ML, Caporali L, et al: Multi-system neurological disease is common in patients with OPAl mutations. Brain 133: 771-786, 2010.

4. Pesch UE, Leo-Kottler B, Mayer S, Jurklies B, Kellner U, Apfelstedt-Sylla E, Zrenner E, Alexander C and Wissinger B: OPA1 mutations in patients with autosomal dominant optic atrophy and evidence for semi-dominant inheritance. Hum Mol Genet 10: 1359-1368, 2001.

5. Schaaf CP, Blazo M, Lewis RA, Tonini RE, Takei H, Wang J, Wong LJ and Scaglia F: Early-onset severe neuromuscular phenotype associated with compound heterozygosity for OPA1 mutations. Mol Genet Metab 103: 383-387, 2011.

6. Bonneau D, Colin E, Oca F, Ferré M, Chevrollier A, Guéguen N, Desquiret-Dumas V, N'Guyen S, Barth M, Zanlonghi X, et al: Early-onset Behr syndrome due to compound heterozygous mutations in OPA1. Brain 137: e301, 2014.

7. Behr C: Die komplizierte, hereditäre-familiäre Optikusatrophie des Kindesalters-ein bisher nicht beschriebener Symptomkomplex. Klin Mbl Augenheilkd 39: 138-60, 1909 (In German).

8. Thomas PK, Workman JM and Thage O: Behr's syndrome. A family exhibiting pseudodominant inheritance. J Neurol Sci 64: 137-148, 1984.

9. Marelli C, Amati-Bonneau P, Reynier P, Layet V, Layet A, Stevanin G, Brissaud E, Bonneau D, Durr A and Brice A: Heterozygous OPA1 mutations in Behr syndrome. Brain 134: e169, 2011; author reply e170.

10. Choi BO, Koo SK, Park MH, Rhee H, Yang SJ, Choi KG, Jung SC, Kim HS, Hyun YS, Nakhro K, et al: Exome sequencing is an efficient tool for genetic screening of Charcot-Marie-Tooth disease. Hum Mutat 33: 1610-1615, 2012.

11. Delettre C, Lenaers G, Griffoin JM, Gigarel N, Lorenzo C, Belenguer P, Pelloquin L, Grosgeorge J, Turc-Carel C, Perret E, et al: Nuclear gene OPA1, encoding a mitochondrial dynamin-related protein, is mutated in dominant optic atrophy. Nat Genet 26: 207-210, 2000.

12. Amati-Bonneau P, Milea D, Bonneau D, Chevrollier A, Ferré M, Guillet V, Gueguen N, Loiseau D, de Crescenzo MA, Verny $\mathrm{C}$, et al: OPA1-associated disorders: Phenotypes and pathophysiology. Int J Biochem Cell Biol 41: 1855-1865, 2009.

13. Lloyd IC, Goss-Sampson M, Jeffrey BG, Kriss A, Russell-Eggitt I and Taylor D: Neonatal cataract: Aetiology, pathogenesis and management. Eye (Lond) 6: 184-196, 1992.

14. Chan WH, Biswas S, Ashworth JL and Lloyd IC: Congenital and infantile cataract: Aetiology and management. Eur J Pediatr 171: 625-630, 2012.

15. Aijaz S, Erskine L, Jeffery G, Bhattacharya SS and Votruba M: Developmental expression profile of the optic atrophy gene product: $O P A 1$ is not localized exclusively in the mammalian retinal ganglion cell layer. Invest Ophthalmol Vis Sci 45: 1667-1673, 2004. 